\title{
Evaluación de la composición del plancton en cuatro lagunas de rebalse del rio Metica (Puerto López, Meta, Colombia)
}

\author{
Evaluation of the plankton composition in four overflow lagoons \\ from Metica river (Puerto Lopez, Meta, Colombia)
}

\author{
Avaliação da composição do plâncton em quatro lagoas de estouro \\ do rio Metica (Puerto Lopez, Meta, Colômbia
}

Lili J. Marciales-Caro ${ }^{1}$, John J. Díaz-Olarte ${ }^{2}$, Pablo E. Cruz-Casallas ${ }^{3}$, Víctor M. Medina-Robles ${ }^{4}$

\begin{abstract}
Bióloga, Esp. Acuicultura, MSc Ciencias Biológicas, Universidad de los Andes, Bogotá, Colombia
Biólogo, Universidad Industrial de Santander, Instituto Colombiano del Petróleo - ICP, Pie de Cuesta, Santander

3 Médico Veterinario Zootecnista, MSc, PhD, Profesor Facultad de Ciencias Agropecuarias y Recursos Naturales, Instituto de Acuicultura de los Llanos - IALL, Villavicencio, Meta - Colombia

4 Médico Veterinario Zootecnista, MSc, Profesor Facultad de Ciencias Agropecuarias y Recursos Naturales, Instituto de Acuicultura de los Llanos - IALL, Villavicencio, Meta - Colombia

* Grupo de Investigación sobre Reproducción y Toxicología de Organismos Acuáticos - GRITOX, Instituto de Acuicultura, Facultad de Ciencias Agropecuarias y Recursos Naturales, Universidad de los Llanos, Villavicencio, Meta-Colombia, Convenio 00325 Unillanos - SENA.

E-mail: lilimarciales@gmail.com
\end{abstract}

Recibido: septiembre 4 de $2012 \quad$ Aceptado: noviembre 29 de 2012

\begin{abstract}
Resumen
Se determinó la composición cualitativa de las comunidades fitoplanctónicas y zooplanctónicas en cuatro lagunas de rebalse, ubicadas en la llanura aluvial del río Metica (Puerto López, Meta), durante las épocas de aguas altas (Junio - Septiembre) y aguas bajas (Noviembre - Diciembre). Entre las cuatro lagunas visitadas se observaron diferencias en las características físicas y químicas del agua, relacionadas con el aporte del cauce principal del rio Metica y de los afluentes provenientes de la sabana. En las lagunas la Virginia y Navajas el aporte principal de agua proviene del río Metica, mientras que la laguna La Campana, por estar aislada, presentó condiciones limnológicas más estables a lo largo del estudio. Por otro lado, la laguna Nare presentó los valores de pH y conductividad más bajos, lo cual puede estar relacionado con la mayor cantidad de aportes de agua de la altillanura que del río Metica. En cuanto a la comunidad fitoplanctónica se determinaron 45 taxones distribuidos en siete grupos algales principales, con mayor representatividad en las Euglenophyceae y Zygnematophyceae. La comunidad zoplanctónica estuvo representada por 27 morfoespecies. En ambos casos, la mayor diversidad fue observada en la laguna Virginia. La riqueza morfoespecífica de las comunidades fito y zooplanctónicas fue mayor en la época de aguas bajas con respecto a la de aguas altas. Las variaciones temporales observadas en los factores abióticos de las lagunas (físicos, químicos, nutrientes) y los cambios en la diversidad plantónica están relacionados con las fluctuaciones hidrológicas.
\end{abstract}

Palabras clave: fitoplancton, nutrientes, orinoquia, plano de inundación, zooplancton. 


\begin{abstract}
We determined phytoplankton and zooplankton cualitative composition in four wetlands, within Metica river floodplain (Puerto López- Meta), during two hydrologic periods: low water and high water periods. About four lakes, we observed differences related with input from the mainstream Metica river and tributaries from savannah to each water body. Nare lake presented $\mathrm{pH}$ and conductivitie values higher than the others lakes, and receives much input from the tributaries from savannah that Metica river. Virginia and Navajas receives much imput from Metica river and Campana lake is isolated and had stable limnological conditions throughout the study. Regarding the phytoplankton community were determined 45 taxa distributed in 7 major algal groups, with greater representation in the Euglenophyceae and Zygnematophyceae. Zooplankton community was represented by 27 morphospecies. Lake Virginia has the highest diversity in both cases. Morphospecific composition of phytoplankton and zooplankton communities was higher in low-water period with respect to high water. Temporal variations observed in abiotic factors (physical and chemical parameters and nutrients) are directly related to hydrological changes, like changes in planktonic diversity.
\end{abstract}

Keywords: floodplain, nutrients, orinoquia, phytoplankton, zooplankton.

\title{
Resumo
}

Foi determinada a composição qualitativa das comunidades do fitoplâncton e zooplâncton em quatro lagoas de várzea, localizadas na planície de inundação do rio Metica (Puerto Lopez, Meta - Colombia), durante os períodos de chuva (junho a setembro) e de seca (novembro e dezembro). Houve diferenças nas características físicas e químicas da água entre as quatro lagoas estudadas, relacionadas com o aporte do canal principal do rio Metica e de outros afluentes provenientes da savana. Nas lagoas Virgínia e Navajas o abastecimento principal de água é do rio Metica, enquanto que a lagoa Campana, sendo isolada, apresentou condições limnológicas mais estáveis ao longo do estudo. Além disso, a lagoa Nare apressentou os menores valores de $\mathrm{pH}$ e condutividade, o que pode estar relacionado com a maior quantidade de água proveniente da savana que do rio Metica. Enquanto à comunidade fitoplânctonica foram determinados 45 táxons distribuidos em sete grupos principais de algas, sendo os mais representativos as Zygnematophyceae e Euglenophyceae. A comunidade zoplanctónica foi representada por 27 morfoespécies. Em ambos os casos, a maior diversidade foi observada na lagoa Virginia. A riqueza morfoespecífica de fito e zooplâncton foi maior durante as águas baixas que nas águas altas. As variações temporais observadas nos fatores abióticos das lagoas (características físicas, químicas e conteudo de nutrientes) e as mudanças na diversidade planctónica estão relacionadas com as flutuações hidrológicas.

Palavras-chave: fitoplâncton, nutrientes, rio Orinoco, planície de inundação.

\section{Introducción}

Los ecosistemas acuáticos de las grandes cuencas de la Orinoquía y la Amazonía tienen alta trascendencia socioeconómica debido a que son el soporte de la actividad pesquera comercial y local (Araujo-Lima et al., 1998), la cual ejerce una presión negativa cada vez más fuerte debido a la sobreexplotación del recurso. Los sistemas acuáticos sobre los cuales se ejerce esta actividad, específicamente en la Orinoquía Colombiana, se ubican en tres subregiones naturales: piedemonte con elevaciones hasta $500 \mathrm{~m}$ ubicado en las proximidades de la Cordillera Oriental; abanicos aluviales con alturas inferiores a los $400 \mathrm{~m}$, compuestas por las vegas de los ríos y sabanas colindantes que se inundan estacionalmente y altillanuras, localizadas en los departamentos de Vichada y Meta que no están sujetas a inundaciones estacionales (Rangel et al., 1995). Tributarios colombianos como los ríos Metica, Meta y Arauca drenan desde las estribaciones de los Andes hacia la región media del Orinoco, originando complejos de humedales continentales caracterizados por presentar tendencia a la oligotrofía, con bajos valores de conductividad eléctrica y $\mathrm{pH}$ con tendencia a la acidez (Galvis et al., 1989; Donato, 1998; Correa et al., 2005; Rivera-Rondón et al., 2010).

Estudios iniciales relacionados con las condiciones físicas y químicas de los humedales asociados a la cuenca media y baja del Orinoco, en territorio Venezolano, sugieren que las características químicas de los ecosistemas a nivel espacial y temporal están determinadas por: 1) el origen geológico de los suelos de las sabanas, ubicadas sobre el escudo Guayanés con suelos muy pobres en nutrientes (Weibezahn et al., 1990; Lewis et al., 1995; Sarmiento \& Pinillos, 2001) 2) fluctuaciones temporales en los niveles de los cauces principales, lo cual origina cambios marcados en la física y química de las lagunas de rebalse como resultado de aportes de nutrientes y sedimentos, y por ello un claro ejemplo en la diversidad biótica presente (Hamilton \& Lewis, 1987; Dezzeo et al., 2000), tal y como se ha evidenciado en los planos de inundación de los ríos Cinaruco (Cotner et al., 2006), Caura (Hamilton \& Lewis, 1990) y el propio Orinoco (Hamilton \& Lewis, 1987) y 3) diferencias en la morfología de la cuenca que pueden determinar características físicas, químicas y ecológicas de los planos de inundación (Hamilton \& Lewis, 1990), 
relacionadas principalmente con las diferencias en material en suspensión y nutrientes (nitrógeno y fósforo).

Las comunidades planctónicas juegan un papel importante en las redes tróficas de estos sistemas acuáticos, siendo la principal fuente de alimento de muchos peces de talla pequeña, y primeros estadios larvales (Galvis et al., 1989; Ramírez-Gil y Ajiaco-Martínez, 2001). En las llanuras aluviales asociadas a los planos de inundación de los ríos, la estructura de estas comunidades se ve afectada principalmente por los aportes diferenciales de nutrientes desde el cauce principal del río, de acuerdo con las fluctuaciones en el nivel del agua, asociada con los periodos hidrológicos conocidos como de aguas bajas y aguas altas (Galvis et al., 1989; Ramírez-Gil y Ajiaco-Martínez, 2001).

El conocimiento de la composición de las comunidades planctónicas es de vital importancia para la prevención y manejo de humedales (Correa et al., 2005; Rivera-Rondón et al., 2010); sin embargo, en la Orinoquia colombiana existen pocos estudios relacionados directamente con la riqueza de especies, así como sobre los cambios ocasionados por la dinámica hidrológica. En la Orinoquia Venezolana, se han realizado estudios de estructura y composición de comunidades planctónicas y su relación con la estacionalidad, observando que el régimen anual en cuanto a abundancia de organismos en suspensión se encuentra claramente relacionado con los eventos hidrológicos (Saunders et al., 1988; Lewis et al., 1990); así mismo, se ha evaluado el efecto de las inundaciones periódicas como factor de disturbio ambiental sobre la dinámica hidrológica y bioquímica del sistema, enfocada hacia la protección de zonas de transición acuático terrestre (San José et al., 2001). En Colombia, si bien están claramente definidas las regiones hidrográficas y complejos de humedales de acuerdo con factores hidrológicos, geomorfológicos, patrones de circulación del agua y fisionomía (Correa et al., 2005), actualmente es poca la información relacionada con las condiciones limnológicas de cuerpos de agua en la región Norte de la Orinoquia Colombiana, específicamente en la cuenca del Río Metica, siendo los estudios y reportes relacionados con las comunidades planctónicas en esta zona escasos (Galvis et al., 1989; González-González et al., 2006).

De acuerdo con lo anterior, el objetivo del presente estudio fue describir el comportamiento de algunos parámetros limnológicos y caracterizar cualitativamente las comunidades planctónicas en cuatro lagunas de rebalse asociadas a las Ilanuras aluviales del río Metica, Municipio de Puerto López, Meta, durante dos periodos hidrológicos (Aguas Altas y Aguas Bajas).

\section{Materiales y métodos}

\section{Área de estudio}

El área de estudio comprendió cuatro lagunas de rebalse (Nare, Virginia, Campana y Navajas), asociadas con las llanuras aluviales del plano de inundación del río Metica, jurisdicción del municipio de Puerto López, departamento del Meta, Colombia (Figura 1). La laguna Nare está ubicada en la margen derecha del rio Metica $\left(3^{\circ} 57^{\prime} 16,3^{\prime \prime} \mathrm{N}-72^{\circ} 59^{\prime} 16,7^{\prime \prime} \mathrm{W}\right)$ y se conecta durante todo el periodo hidrológico con el río por medio de un pequeño caño, sin existir un aislamiento total del río en la época seca (Figura 1). Adicionalmente, la laguna recibe aportes del caño Nare $\left(3^{\circ} 58^{\prime} 23,8^{\prime \prime}\right.$ $\left.\mathrm{N}-72^{\circ} 59^{\prime} 24,3^{\prime \prime} \mathrm{W}\right)$ proveniente de la altillanura.

El complejo lagunar La Virginia, ubicado en la margen derecha del río Metica ( $3^{\circ} 59^{\prime} 24,4^{\prime \prime} \mathrm{N}-72^{\circ} 57^{\prime} 39,4^{\prime \prime}$ $W)$, el cual se encuentra aislado del río en época seca, separado aproximadamente $800 \mathrm{~m}$ de la rivera. En época de lluvias su plano de inundación recibe aportes de otras lagunas (Figura 1). Este complejo es el resultado de la acción de la dinámica fluvial reciente del río Metica, el cual inunda una parte de bosque. La laguna La Campana, ubicada en la margen izquierda del curso principal del río Metica ( $\left.4^{\circ} 1^{\prime} 4,72^{\prime \prime} \mathrm{N}-72^{\circ} 5,0^{\prime} 11^{\prime \prime}\right)$, tiene forma de herradura (Figura 1) y se define como una madrevieja antigua del río, la cual fue aislada por completo del cauce principal como consecuencia de la dinámica fluvial. Esta laguna tiene contacto con el río solo en época de aguas altas, cuando su cauce llega hasta la laguna. Tiene un espejo de agua bien definido y permanente en época de aguas bajas.

Finalmente, la laguna Navajas, la cual se encuentra localizada en la margen derecha del curso principal del rio Metica $\left(4^{\circ} 00^{\prime} 32,5^{\prime \prime} \mathrm{N}-72^{\circ} 56^{\prime} 48,1^{\prime \prime} \mathrm{W}\right)$, y al igual que la laguna Nare, tiene un caño que la conecta con el río solo en época de aguas altas (Figura 1).

\section{Variables físicas y químicas}

Se seleccionaron en total ocho estaciones en las cuatro lagunas, ubicadas en la zona limnética, definiendo dos estaciones en cada cuerpo de agua. Se realizaron muestreos mensuales incluyendo tanto la época de aguas altas o época de Lluvias (Junio - Septiembre 2008), como de aguas bajas o época seca (Noviembre - Diciembre 2008), para un total de 7 jornadas de muestreo.

Con el fin de determinar las características limnológicas principales de los hábitats muestreados, en cada estación a nivel superficial se midió transparencia (dis- 

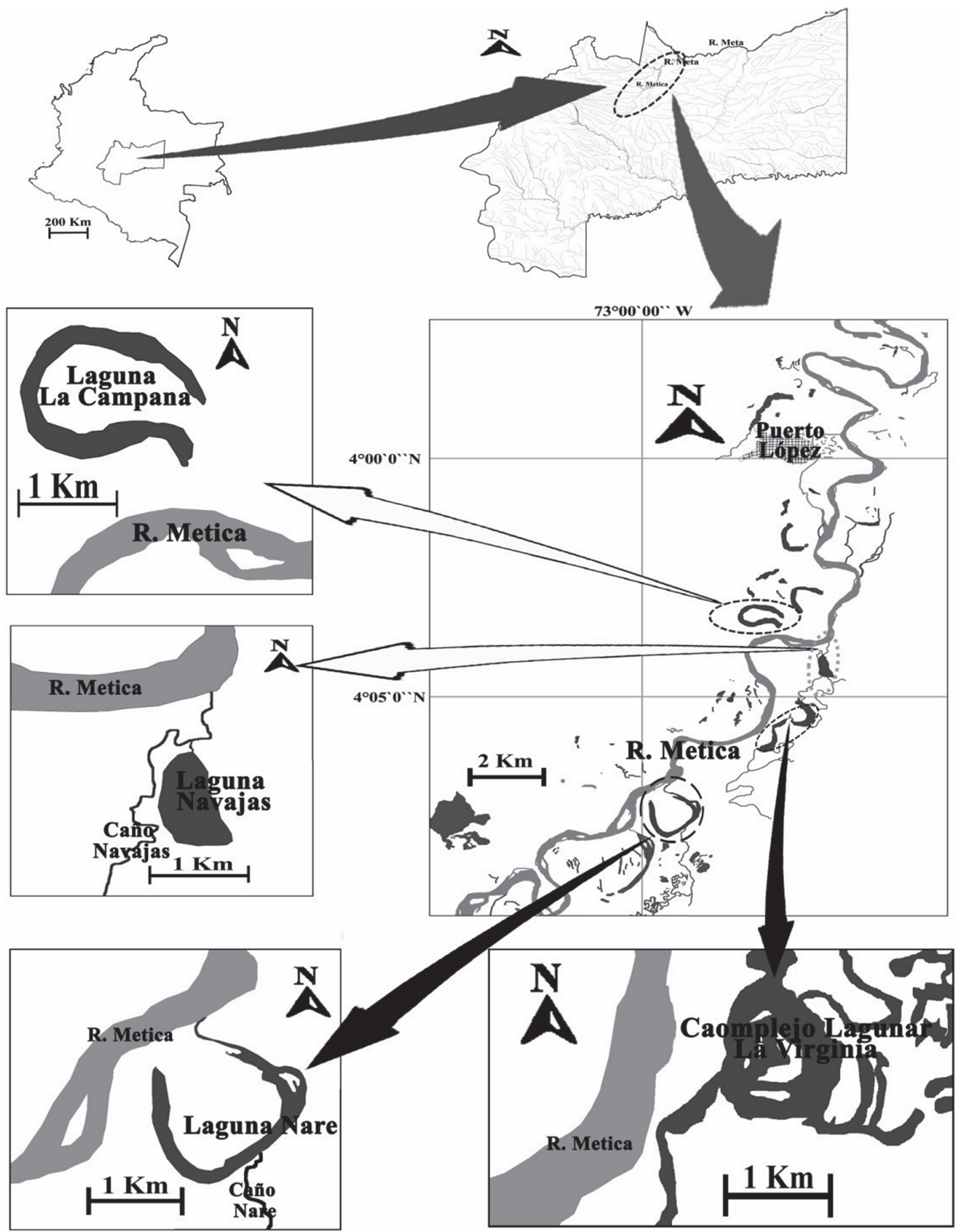

Figura 1. Ubicación de las lagunas estudiadas con respecto al curso principal del río Metica (Puerto López, Meta, Colombia). 
co Secchi), temperatura $\left({ }^{\circ} \mathrm{C}\right)$, oxígeno disuelto $(\mathrm{mg} / \mathrm{L})$, $\mathrm{pH}$, sólidos totales disueltos TDS ( $\mathrm{mg} / \mathrm{L})$ y conductividad $(\mu \mathrm{s} / \mathrm{cm})$, utilizando una sonda multiparamétrica (YSI Professional Plus, USA). Se tomaron muestras a nivel superficial, para determinar las concentraciones de amonio $\left(\mathrm{NH}_{4}^{+}\right)$, nitrato $\left(\mathrm{NO}_{3}^{-}\right)$y nitrito $\left(\mathrm{NO}_{2}^{-}\right)$. Estos últimos análisis fueron realizados de acuerdo con los procedimientos establecidos por la American Public Health Association (APHA) para la determinación de nutrientes en cuerpos de agua continentales (APHA, 2005). Las variables abióticas como oxígeno, temperatura, conductividad y nutrientes se analizaron mediante la técnica de ordenación tipo componentes principales ACP (Legendre \& Legendre, 1998). Todas
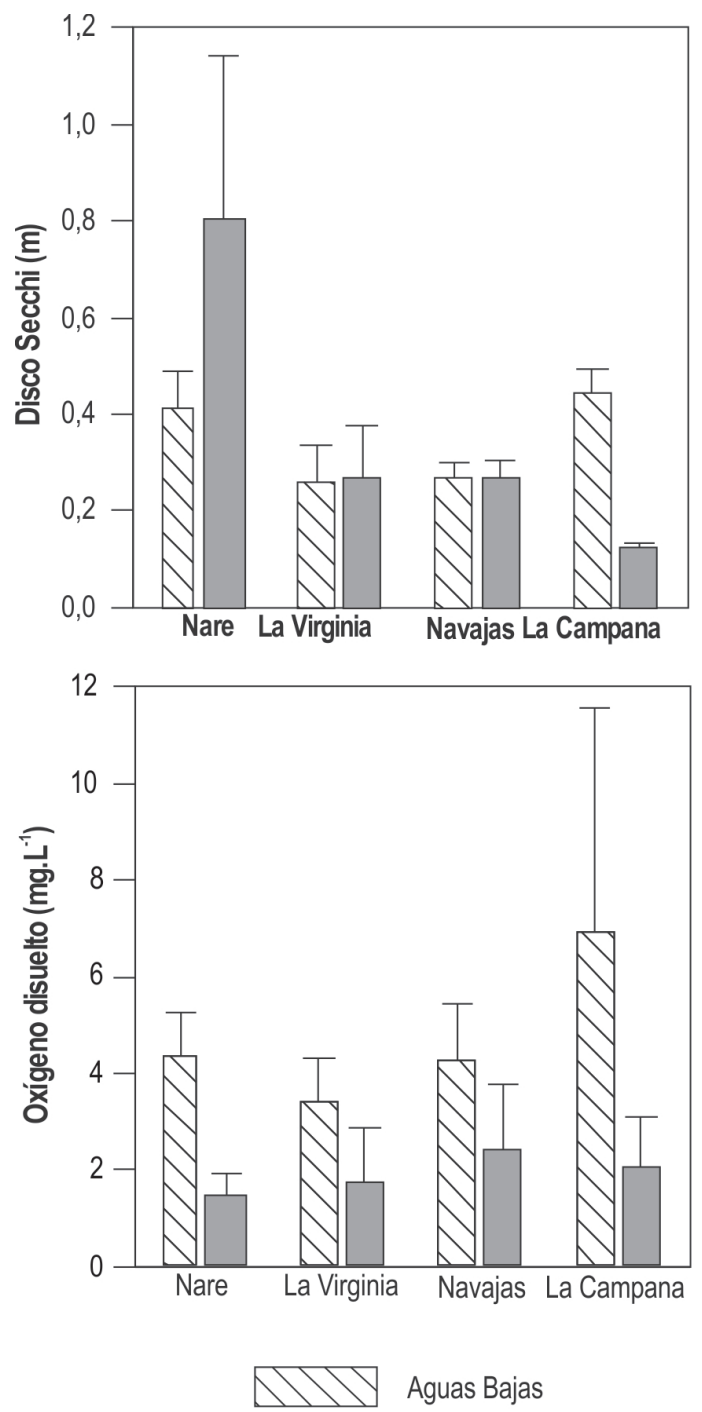

las variables físico-químicas fueron expresadas como media \pm desviación estándar (SD).

\section{Muestras biológicas}

En cada estación de muestreo se tomaron muestras biológicas cualitativas para estudiar la composición de la comunidad planctónica. Las muestras fueron colectadas a nivel superficial, entre las 8:00 y 11:00 horas, realizando arrastres horizontales con mallas de 25 y $60 \mu \mathrm{m}$ para comunidades fitoplanctónicas y zooplanctónicas, respectivamente. Las muestras fueron fijadas con solución Transeau (Agua destilada, alcohol y formol en proporción 6:3:1) en una proporción 1: 1 .
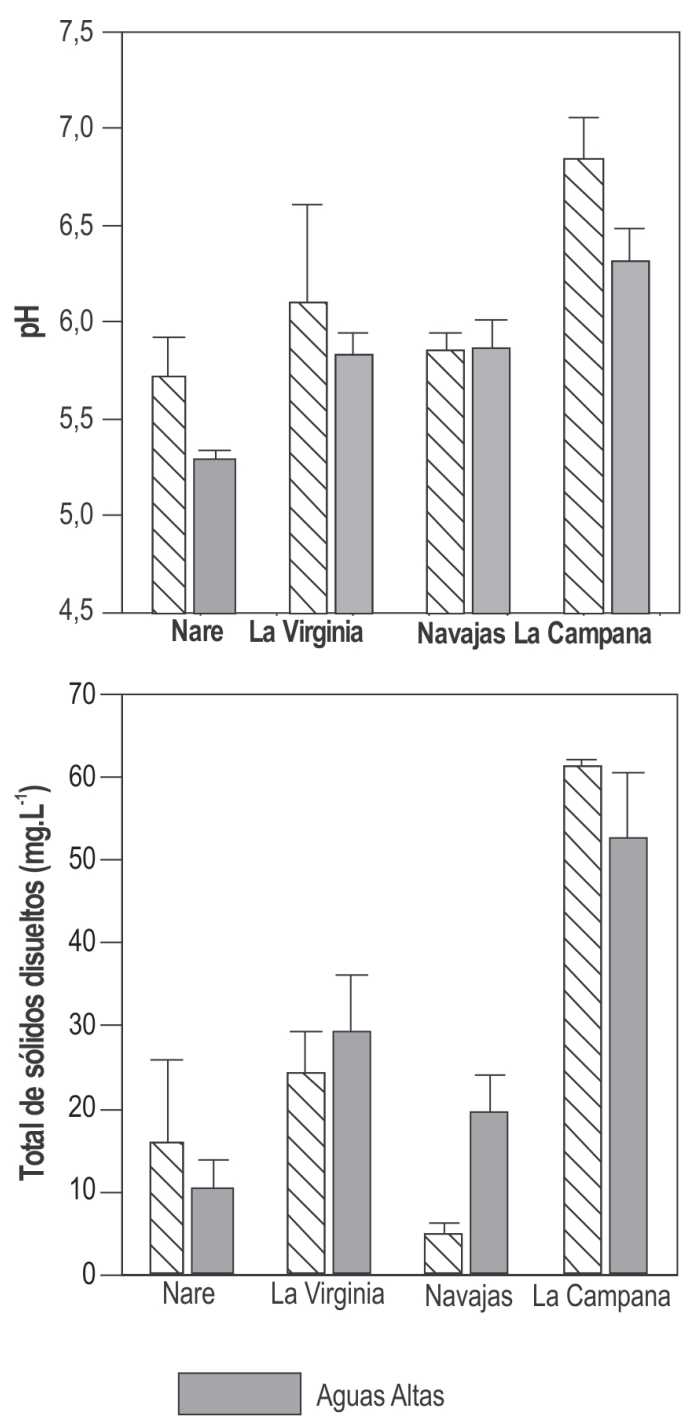

Figura 2. Principales variables físicas y químicas medidas en cada una de las lagunas de rebalse asociadas al Río Metica. Valores mostrados como media \pm SD $(n=7)$ 

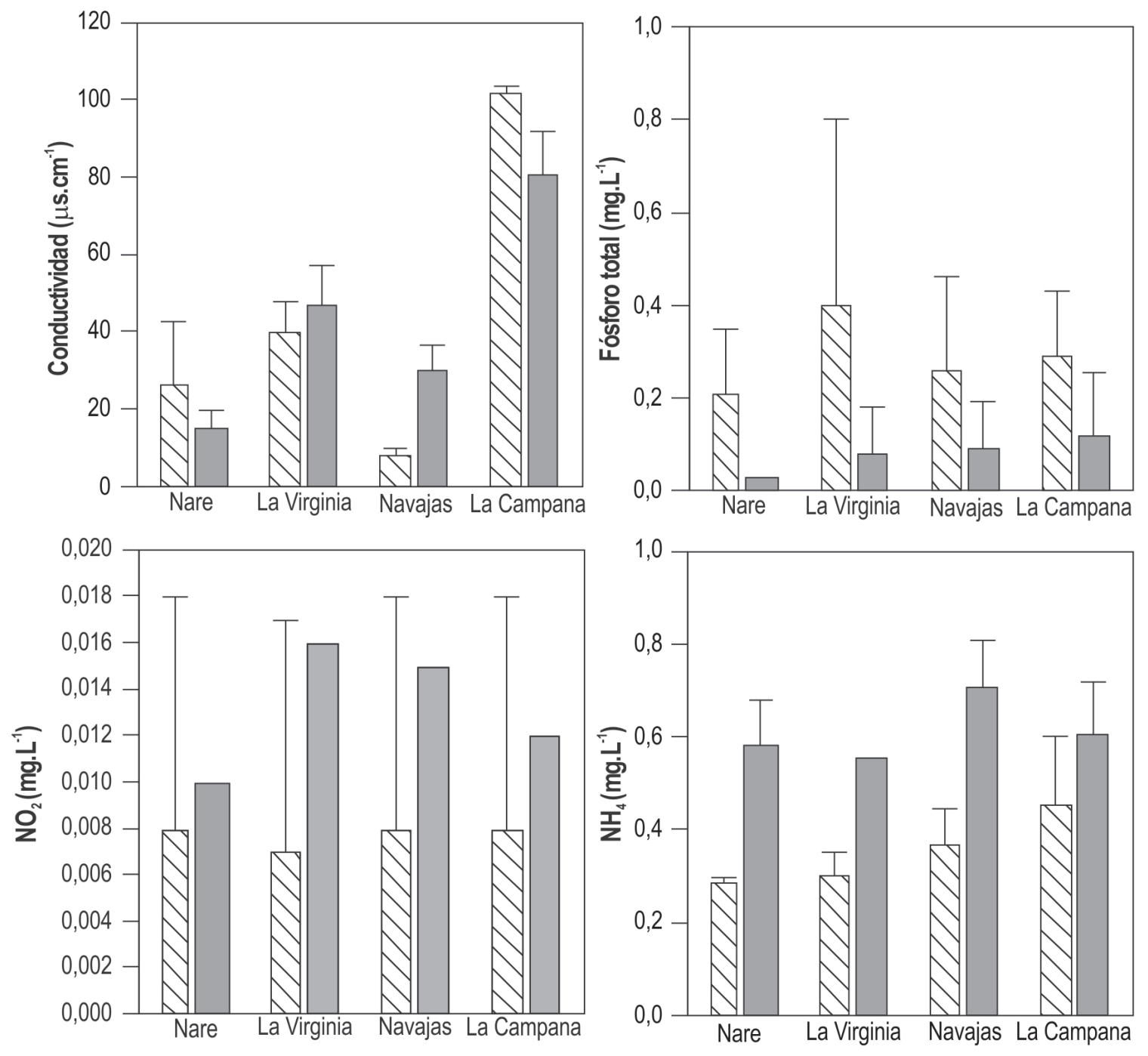

M11. Aguas Bajas

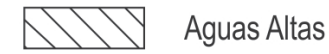

Figura 3. Valores de conductividad eléctrica y de contenido de nutrientes medidos en cada una de las lagunas de rebalse asociadas al Río Metica. Datos mostrados como media \pm SD $(n=7)$.

Posteriormente en el laboratorio se realizó la determinación de los organismos del componente fitoplanctónico por medio de claves taxonómicas específicas para cada grupo hasta el nivel de género o especie (Bicudo et al. 1982, 1984; Coesel 1983, 1985, 1991; Comas, 1996; Croasdale et al., 1983; Tell \& conforti, 1986; Pestalozzi, 1955; Prescott et al., 1975, 1977, 1981, 1982 y Sala et al., 2002). Para las comunidades zooplanctónicas se usaron claves taxonómicas específicas de Elmoor-Loureiro (1997) y Paggi \& Paggi (1990).

\section{Resultados}

\section{Variables físicas y químicas}

Las figuras 2 y 3 resumen las variables físicas y químicas medidas In Situ en las cuatro lagunas, tanto en época de aguas bajas como durante el periodo de aguas altas. Las máximas fluctuaciones se presentaron en aguas altas en las variables de $\mathrm{pH}$, conductividad, nitrito, amonio y fosforo total, observándose los valores más bajos en las lagunas con mayores aportes de afluentes provenientes de la altillanura, como es el 


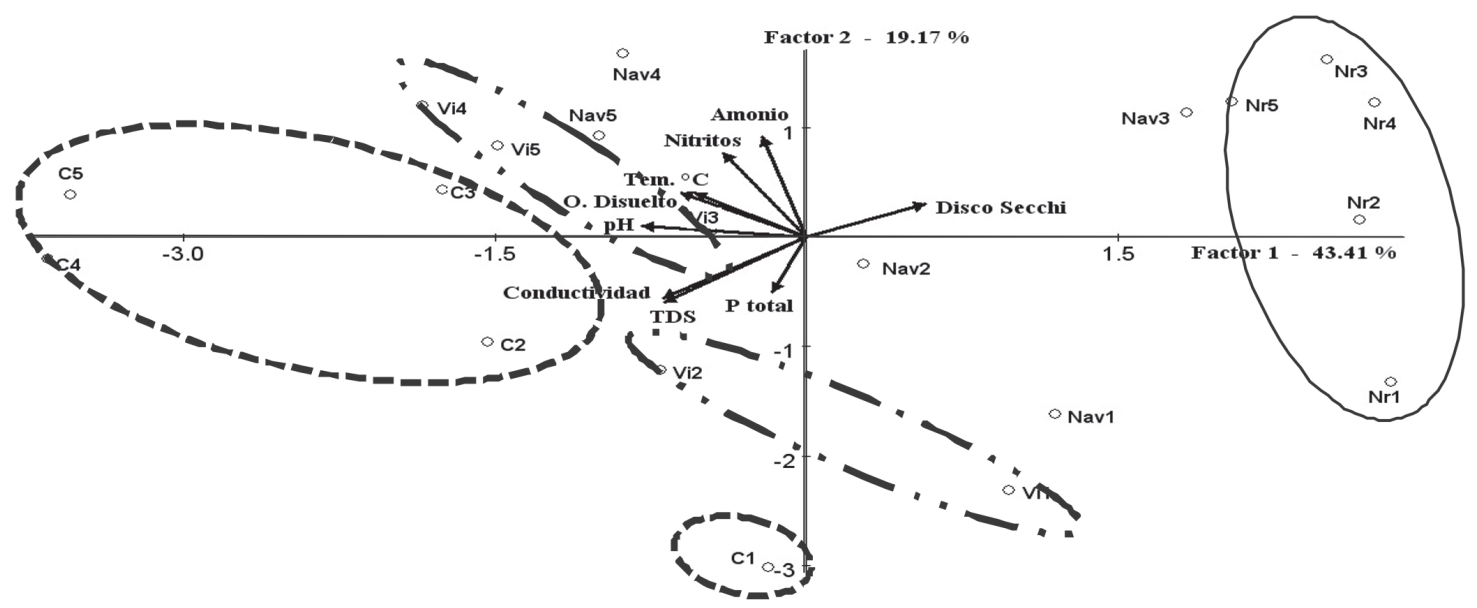

Figura 4. Posición de las variables abióticas para las cuatro lagunas en el espacio dimensionado para los dos componentes principales, según Análisis de Componentes Principales (ACP). Las variables ambientales se indican por las flechas en el espacio de los dos primeros componentes principales. (1) Junio 2008, (2) Agosto 2008, (3) Septiembre 2008, (4) Noviembre - Diciembre 2008 y (5) Diciembre 2008. C: La Campana, Vi: La Virginia, Nr: Nare y Nav: Navajas.

caso de la laguna Nare. Por otro lado, la laguna La Campana permanece la mayor parte del tiempo aislada de los aportes del río Metica, el cual la inunda sólo en la cota máxima; sin embargo, este cuerpo de agua presentó los valores más altos de conductividad y de total de sólidos disueltos.

El análisis de componentes principales (ACP) de estos parámetros muestra grandes cambios según el régimen hidroclimático. Los dos primeros factores (Ejes) explican el $62.6 \%$ de la variación total de los datos (Figura 4). El primer factor tiene una correlación positiva con la transparencia y una correlación negativa con el $\mathrm{pH}$, conductividad, oxígeno disuelto, sólidos totales disueltos y temperatura; el segundo factor se correlaciona positivamente con las concentraciones de nitritos y amonio y negativamente con el fósforo total (Figura 4). En la representación gráfica del plano de ordenación se observan tres grupos bien definidos según las condiciones limnológicas de cada laguna, separando en el caso de la laguna Virginia la época de aguas bajas de la época de aguas altas. El primer grupo está formado por el Lago Nare, agrupando tanto los muestreos de

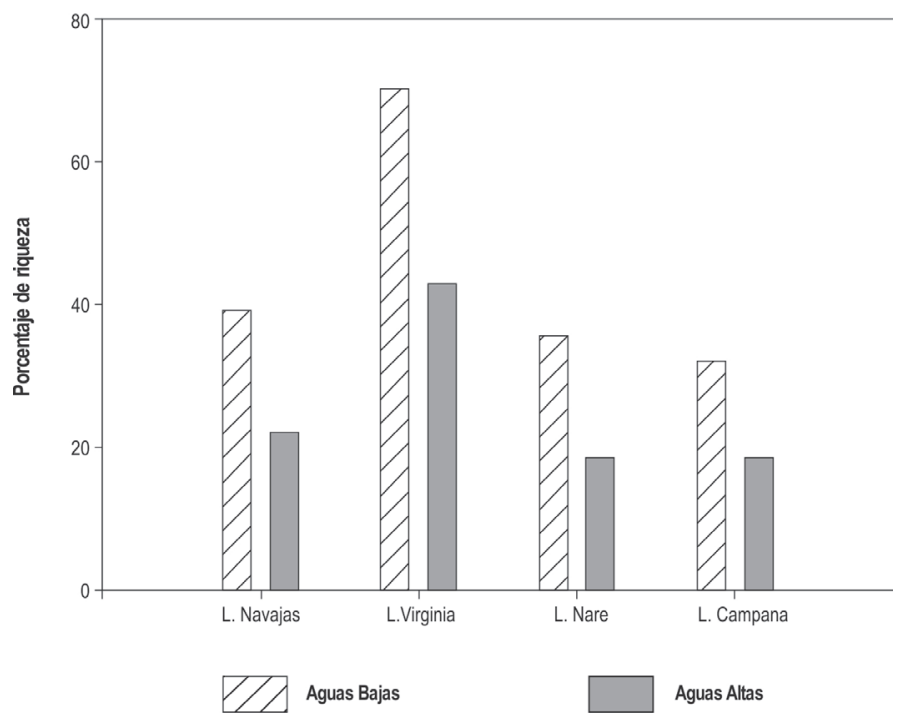

Figura 5. Porcentajes de Riqueza morfoespecífica de la comunidad fitoplanctónica, presente en las cuatro lagunas de rebalse asociadas al Río Metica, para las épocas de aguas bajas y aguas altas. 


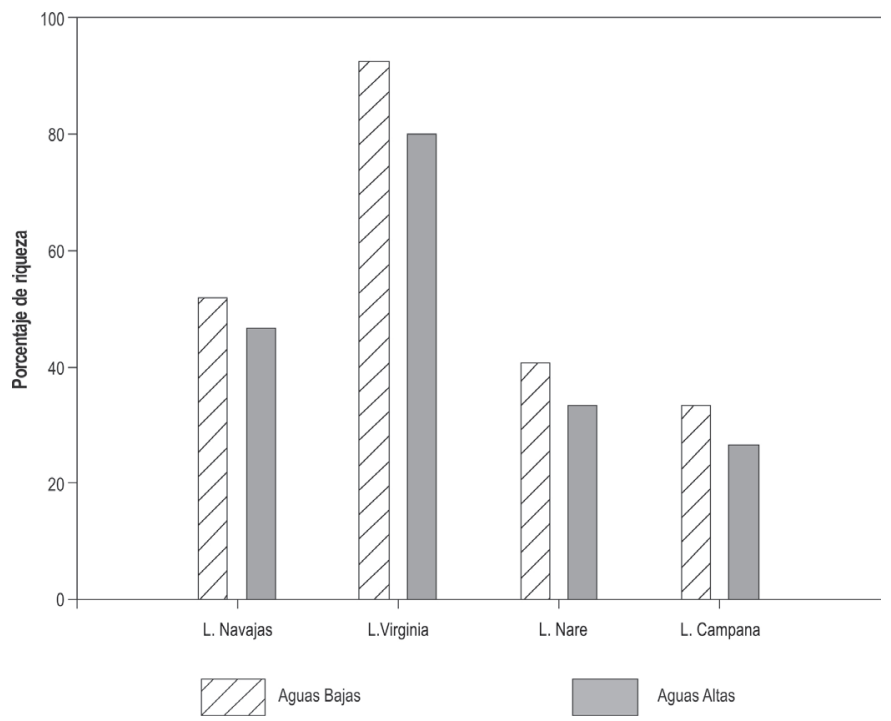

Figura 6. Porcentajes de Riqueza morfoespecífica de la comunidad zooplanctónica, presente en las cuatro lagunas de rebalse asociadas al Río Metica, para las épocas de aguas bajas y aguas altas.

Tabla 1. Lista de morfoespecies fitoplanctónicas determinadas para las lagunas de rebalse asociadas al río Metica. AA: Aguas Altas, AB: Aguas Bajas. ( $\mathrm{X}=$ presencia $)$.

\begin{tabular}{|c|c|c|c|c|c|c|c|c|c|}
\hline \multirow{3}{*}{ Clase } & \multirow{3}{*}{ Géneros } & \multicolumn{8}{|c|}{ Lagunas } \\
\hline & & \multicolumn{2}{|c|}{ Navajas } & \multicolumn{2}{|c|}{ Virginia } & \multicolumn{2}{|c|}{ Nare } & \multicolumn{2}{|c|}{ Campana } \\
\hline & & AA & AB & AA & $A B$ & AA & $A B$ & AA & $\mathbf{A B}$ \\
\hline Bacillariophyceae & Diatoma sp 1 & $x$ & & & & & & & \\
\hline Bacillariophyceae & Eunotia sp 1 & & & $x$ & $x$ & & & & \\
\hline Bacillariophyceae & Gomphonema sp 1 & 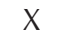 & & & & & & $x$ & \\
\hline Bacillariophyceae & Rhizolenia sp 1 & & & & & $x$ & & & \\
\hline Bacillariophyceae & Stenopterobia sp 1 & $x$ & & & & $x$ & & & \\
\hline Bacillariophyceae & Stenopterobia sp2 & & & & & & $x$ & & \\
\hline Bacillariophyceae & Pinularia sp 1 & & & $x$ & & & & & \\
\hline Bacillariophyceae & Surirella sp 1 & & & & & $x$ & $x$ & & \\
\hline Bacillariophyceae & Nitszchia sp1 & & & & $x$ & & & & \\
\hline Cyanophyceae & Pediastrum sp 1 & & & $x$ & & & & $x$ & \\
\hline Cyanophyceae & Scenedesmus sp 1 & & & $x$ & & & & & \\
\hline Cyanophyceae & Oscillatoria sp 1 & $x$ & & & & & & & \\
\hline Cyanophyceae & Phormidium sp 1 & & & $x$ & & & & & \\
\hline Cyanophyceae & Anabaena sp1 & & & & & & & & $x$ \\
\hline Cyanophyceae & Anabaena sp2 & & $x$ & & & & & & \\
\hline Crysophyceae & Dynobrion sp1 & & & & $x$ & & & & \\
\hline Euglenophyceae & Euglena sp 1 & $x$ & & & $x$ & & & $x$ & \\
\hline Euglenophyceae & Lepocinclis sp 1 & $X$ & & & & & & $X$ & \\
\hline
\end{tabular}




\begin{tabular}{|c|c|c|c|c|c|c|c|c|c|}
\hline \multirow{3}{*}{ Clase } & \multirow{3}{*}{ Géneros } & \multicolumn{8}{|c|}{ Lagunas } \\
\hline & & \multicolumn{2}{|c|}{ Navajas } & \multicolumn{2}{|c|}{ Virginia } & \multicolumn{2}{|c|}{ Nare } & \multicolumn{2}{|c|}{ Campana } \\
\hline & & AA & $A B$ & AA & $A B$ & AA & $A B$ & AA & $A B$ \\
\hline Euglenophyceae & Lepocinclis sp 2 & & & $\mathrm{x}$ & & & & & \\
\hline Euglenophyceae & Lepocinclis sp 3 & & & $x$ & & & & & \\
\hline Euglenophyceae & Phacus longicauda & & & $x$ & & & & & \\
\hline Euglenophyceae & Phacus sp 1 & $\mathrm{x}$ & & & $x$ & & & $\mathrm{x}$ & \\
\hline Euglenophyceae & Phacus sp 2 & & & $\mathrm{x}$ & & & & $x$ & \\
\hline Euglenophyceae & Phacus sp 3 & & & & & $\mathrm{x}$ & & & \\
\hline Euglenophyceae & Strombomonas sp 1 & $x$ & & & $x$ & & & & \\
\hline Euglenophyceae & Strombomonas sp 2 & & & & $x$ & & & & \\
\hline Euglenophyceae & Trachelomonas sp 1 & $\mathrm{x}$ & $\mathrm{x}$ & & $x$ & & & & $x$ \\
\hline Euglenophyceae & Trachelomonas sp 2 & & $\mathrm{x}$ & & $x$ & & & & \\
\hline Euglenophyceae & Trachelomonas sp 3 & & $x$ & & $x$ & & $x$ & & $x$ \\
\hline Euglenophyceae & Trachelomonas sp 4 & & & & $x$ & & & & $x$ \\
\hline Chlorophyceae & Eudorina sp 1 & $\mathrm{x}$ & & $x$ & $\mathrm{X}$ & & & $\mathrm{x}$ & \\
\hline Chlorophyceae & Pandorina sp 1 & $x$ & & $\mathrm{x}$ & & $x$ & & & \\
\hline Zygnematophyceae & Closterium sp 1 & & & & $x$ & $x$ & & & \\
\hline Zygnematophyceae & Closterium sp 2 & & & & $\mathrm{x}$ & & & & \\
\hline Zygnematophyceae & Hyalotheca sp 1 & & & & & $\mathrm{x}$ & $\mathrm{x}$ & & \\
\hline Zygnematophyceae & Mougeotia sp 1 & & & $x$ & & $x$ & & $x$ & \\
\hline Zygnematophyceae & Micrasterias sp 1 & & & & & $x$ & & $x$ & \\
\hline Zygnematophyceae & Staurodesmus sp1 & & & & $x$ & & & & \\
\hline Zygnematophyceae & Staurodesmus sp2 & & & & $x$ & & & & \\
\hline Zygnematophyceae & Staurodesmus sp3 & & $x$ & & & & & & \\
\hline Zygnematophyceae & Actinotaenium sp1 & & & & $x$ & & & & \\
\hline Zygnematophyceae & Cosmarium sp1 & & & & $\mathrm{x}$ & & & & \\
\hline Zygnematophyceae & Staurastrum sp1 & & & & $\mathrm{x}$ & & & & \\
\hline Zygnematophyceae & Bambusina sp1 & & & & & & $x$ & & \\
\hline Zygnematophyceae & Sprirogyra sp 1 & & & & & $x$ & & & \\
\hline
\end{tabular}

aguas bajas como de aguas altas, explicado positivamente por la variable transparencia, y negativamente por el $\mathrm{pH}$, la conductividad y sólidos totales disueltos. El segundo grupo se encuentra formado por la Laguna Virginia, con dos subgrupos de acuerdo con la época de muestreo; Para la época entre junio y agosto (Aguas altas), se encuentra una correlación positiva con la variable fósforo total, mientras que para la época de aguas bajas (Septiembre - Diciembre), la correlación positiva se presenta con las variables $\mathrm{pH}$ y oxígeno disuelto. El tercer y último grupo lo conforma la laguna Campana, agrupando la totalidad de los muestreos, con correlación positiva con las variables de conductividad y $\mathrm{pH}$, y negativa con la transparencia.

\section{Fitoplancton}

Esta comunidad no fue muy diversa en los sistemas naturales muestreados, debido a la baja concentración 
Tabla 2. Lista de morfoespecies zooplanctónicas identificadas para las lagunas de rebalse asociadas al río Metica.

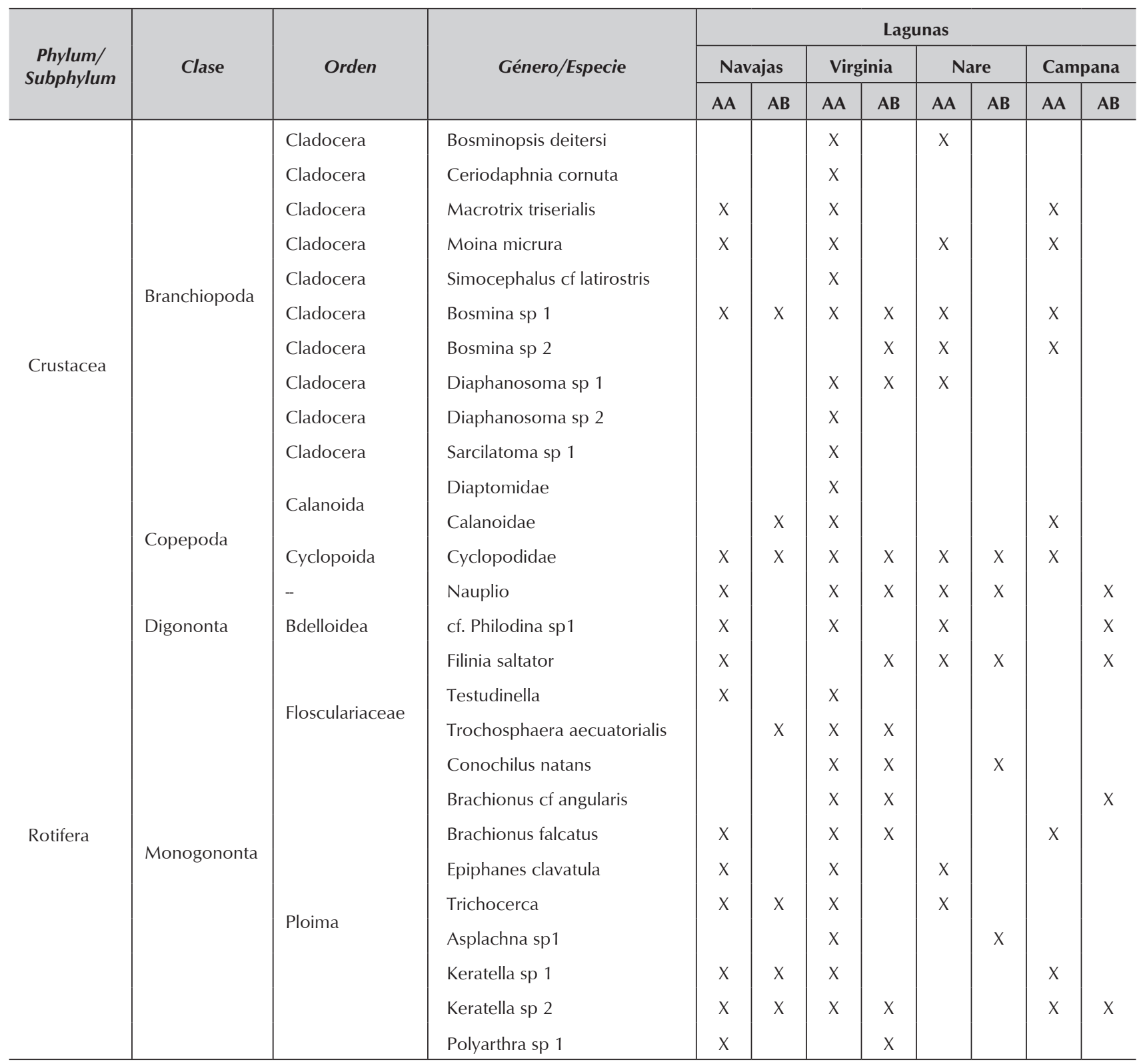

AA: Aguas Altas, AB: Aguas Bajas.

de nutrientes en el medio. Sin embargo, se identificaron algunos individuos pertenecientes a la clase de las Euglenales, representada por los géneros: Phacus $s p$., Euglena $s p$, Trachelomonas $s p$, Strombomonas sp y Lepocinclis $s p$.

En la laguna La Virginia, se encontraron algunas desmidias como Closterium, Micrasterias sp., Hyaloteca sp., Cosmarium sp. Estos géneros son típicos de sitios con presencia de macrófitos, tal y como ocurre en laguna La Virginia. En la tabla 3 se muestra en detalle la dis- tribución de estos individuos en época de aguas bajas y aguas altas. Para la época de aguas altas, disminuyó la riqueza morfoespecífica en las lagunas (Figura 5), como resultado del efecto de dilución producido por el aumento de nivel en cada cuerpo de agua. Sin embargo, se mantuvieron tendencias en cuanto a las lagunas con la mayor riqueza, y la distribución de esta en los grandes grupos taxonómicos. Entre morfoespecies se determinaron 45 taxones distribuidos en siete grupos algales principales. La laguna que presentó la 
mayor riqueza fue la Virginia, con 29 morfoespecies fitoplanctónicas.

\section{Zooplancton}

Esta comunidad se encontró representada principalmente por rotíferos de los géneros: Keratella sp., Polyarthra sp; los cladóceros Bosmina sp, Sarcilatoma sp y los copépodos pertenecientes a las familias Cyclopoidae, Diaptomidae. En la tabla 4 se muestra un resumen de la representación en cada lago de los diferentes organismos para la época de aguas bajas y aguas altas. El comportamiento de los valores de riqueza frente al cambio de estación climática es similar al que ocurre en la comunidad fitoplanctónica, disminuyendo su riqueza en la época de aguas altas en las lagunas estudiadas, debido al efecto de dilución en cada cuerpo de agua (Figura 6). Entre morfoespecies se determinaron 27 taxones distribuidos en 13 rotíferos, 10 cladóceros y 4 copépodos, incluyendo como una morfoespecie los copépodos en estadio de nauplio. La laguna que presentó la mayor riqueza fue la Virginia, con 27 morfoespecies zooplanctónicas, es decir, la totalidad de las morfoespecies determinadas fueron encontradas en la laguna La Virginia, en alguna de las dos épocas de muestreo.

\section{Discusión}

Las variables físicas y químicas evaluadas en el presente estudio permiten evidenciar diferencias entre las lagunas muestreadas, relacionadas con el nivel de conectividad con el cauce principal del río Metica y los aportes de afluentes originados en la altillanura, caracterizados por $\mathrm{pH}$ ácidos y bajos contenidos de nutrientes (Galvis et al., 1989; Donato, 1998; Correa et al., 2005; Rivera-Rondón et al., 2010), lo cual está estrechamente relacionado con la naturaleza de su origen en suelos del escudo Guayanés, muy pobres en estos elementos químicos (Sarmiento y Pinillos, 2001; Berrio et al., 2002; Cortés, 2004; Romero et al., 2004).

Las condiciones de $\mathrm{pH}$ ácido y de alta transparencia observadas en la laguna Nare indican que los aportes son principalmente del caño Nare, aún en época de aguas altas, contrario a lo que se puede observar en la laguna La Campana, la cual se encuentra completamente aislada del cauce principal del río, con conductividades similares e incluso más altas que las observadas en el cauce principal del río. Esta laguna es resultado de la dinámica fluvial del río Metica, siendo un rezago del cauce principal del río, convertido en una madrevieja, lo cual explica sus condiciones de $\mathrm{pH}$ y conductividad similares a las del río; adicional- mente, recibe aportes del río únicamente en época de aguas altas cuando inunda la planicie aluvial que se encuentra en medio del río y la laguna; su condición aislada le permite mantener condiciones limnológicas estables a lo largo del periodo hidrológico. Las concentraciones de fósforo son menores en la laguna Nare, la cual recibe el mayor aporte de afluentes de altillanura. Temporalmente se presentan algunas variaciones en cuanto a los nutrientes, relacionadas con las fluctuaciones hidrológicas. Igualmente, la caracterización de las condiciones físicas y químicas de estos sistemas de manera continua y más amplia, tanto espacial como temporalmente permitiría una clasificación de los tipos de agua, homologable con las aguas blancas y negras de la Amazonía (Duque 1997, Caro et al. 2010).

En cuanto a las comunidades fitoplanctónicas, se encontró en las cuatro lagunas una baja variedad taxonómica, comparado con sistemas con condiciones limnológicas similares, como los sistemas amazónicos (Marciales-Caro, 2006; Núñez y Duque, 2001; Núñez, 2004; Torres-Bejarano, 2006). Estos resultados concuerdan con los reportados por González- González (2006) para el sistema lagunar de Mata-Mata en el municipio de Puerto López.

Se encontró como grupo predominante en riqueza a las Euglenales, para las lagunas la Virginia, Navajas y la Campana. El crecimiento de estos grupos se ve favorecido en ambientes ricos en materia orgánica (Conforti \& Nudelman, 1994; Reynolds, 2006; Reynolds et al., 2002), por lo cual estos valores de riqueza aumentaron a medida que se presentó la época de aguas bajas. Además, es conocido que este grupo suele ser el más abundante en ecosistemas de tierras bajas de la región amazónica (Gómez, 2008; Palma, 2011).

En el caso de la laguna Nare, la baja variedad taxonómica con respecto a las demás lagunas y la presencia de géneros del grupo de las Zygnematophyceae, denota su tendencia a la oligotrofía, resultados similares a los reportados para Mateyuca y Floramarilla por Coesel $(1988,1992)$ y Losada (1992) y para otros humedales en la Orinoquía pertenecientes a planos de inundación (González et al., 2006; Rivera et al., 2010).

En cuanto al zooplancton la composición taxonómica fue baja, dominada principalmente por rotíferos de hábitos planctónicos como Filinia sp., Brachionus sp., Conochilus sp., y Polyarthra sp. Existen cambios en la diversidad de organismos planctónicos a lo largo del periodo hidrológico, reflejado en la disminución de la riqueza de organismos en época de aguas altas, acorde con lo reportado para la laguna la Orsinera ubicada 
en el plano de inundación del rio Orinoco (Twombly y Lewis, 1987), el Río Orinoco (Lewis et al., 1990) y para el Río Caura (Saunders y Lewis, 1988) en la Orinoquia Venezolana.

Las comunidades planctónicas para las lagunas del rebalse del río Metica, cuentan con una variedad taxonómica baja, siendo las Euglenophyceae las de mayor variedad taxonómica, en especial para las lagunas La Virginia, Navajas y La Campana, y reportando adicionalmente grupos de microalgas caracteristicos de ecosistemas con pH acido, como lo son las Zygnematophyceae.

Con el presente trabajo, se hizo evidente la falta de información sobre comunidades planctónicas en la región norte de la Orinoquia Colombiana; por lo tanto, el presente estudio aporta información base sobre las condiciones limnológicas y la diversidad en sistemas de llanura aluvial asociados al plano de inundación del río Metica. Es necesario el complemento con datos de abundancias y biomasas que permitan establecer la dinámica estacional y el flujo de energía en estos ecosistemas. Adicionalmente, el monitoreo constante de estas comunidades permitirá establecer a tiempo disturbios ambientales que puedan afectar el correcto funcionamiento de los ecosistemas.

\section{Agradecimientos}

El presente trabajo fue elaborado en el marco del proyecto "Producción de alimento vivo a partir de comunidades zooplanctónicas nativas para su utilización como primera alimentación en silúridos" convenio 00325, Cofinanciado por el Servicio Nacional de Aprendizaje SENA y la Universidad de los Llanos, desarrollado por el grupo de Investigación GRITOX en el Instituto de Acuicultura de la Universidad de los Llanos - IALL. Los autores agradecen a los aprendices SENA Orlando Pardo, Hugo Ibáñez y Pablo Luis Soto, técnicos profesionales en Acuicultura, por su valiosa colaboración en los trabajos de campo y de laboratorio.

\section{Referencias}

APHA, AWWA, WEF. 2005. Standard methods for the examination of water and wastewater. 21 th ed. Washington, D.C.

Araujo-Lima CA, Goulding M, Fosberg B, Victoria R, Martinelli L. The Economic Value of the Amazonian flooded forest from a fisheries perspective. Verh Internat Verein Limno, 1998; 26:21772179.

Berrio JC, Hooghiemstra $\mathrm{H}$, Behling $\mathrm{H}$, Botero $\mathrm{P}$, van der Borg $\mathrm{K}$. Late-Quaternary savanna history of the Colombian Llanos Orientales from Lagunas Chenevo and Mozambique: a transect synthesis. The Holocene 2002; 12 (1): 35-48.
Bicudo C, Samanez I. 1984. Desmidioflórula paulista III: generos Bambusina, Desmidium, Groenbladia, Hyalotheca, Onychonema, Phymatodocis, Spondylosium y Teilingia. J. Cramer.

Bicudo C, Sormus L. 1982. Desmidioflórula paulista II: Micrasterias C. Agardh ex Ralfs. Cramer.

Caro C, Trujillo F, Suárez CF, Usma JS. 2010. Evaluación y oferta regional de humedales de la Orinoquia: contribución a un sistema de clasificación de ambientes acuáticos. Capítulo 11-7. Pp. 434-447. En: Lasso, C. A., J. S. Usma, F. Trujillo y A. Rial (eds.). 2010. Biodiversidad de la cuenca del Orinoco: bases científicas para la identificación de áreas prioritarias para la conservación y uso sostenible de la biodiversidad. Instituto de Investigación de Recursos Biológicos Alexander von Humboldt, WWF Colombia, Fundación Omacha, Fundación La Salle e Instituto de Estudios de la Orinoquia (Universidad Nacional de Colombia). Bogotá, D. C., Colombia.

Coesel P. 1983. De desmidiaceeën van nederland -Sieralgen-. Deel 2. Fam. Closteriaceae. Stiching Uitgeverij koninklijke Nederlandse Naturhistorische Verening.

Coesel P. 1985. De desmidiaceeë van nederland. Dell 3 fam. Desmidiaceae (1). Uitgeverij koninklijke Nederlandse Naturhistorische Verening.

Coesel P. 1991. De desmidiaceeën van Nederland. Deel 4 Fam. Desmidiaceae (2). Stichting Uitgeverij koninklijke Nederlandse Natuurhistorische Vereniging.

Coesel P. Desmid assemblages along altitude gradients in Colombia. Nova Hedwigia 1992; 55 (3-4): 353-366.

Coesel P, Duque S, Arango G. Distributional patterns in some neotropical desmid species algae, chlorophyta) in relation to migratory bird routes. Rev hidrobiol trop 1988; 21 (3): 197-205

Comas A. 1996. Las Chlorococcales dulciacuícolas de Cuba. Primer edición, J Cramer.

Conforti V, Nudelman A. Ultrastructure of the Lorica of Trachelomonas Ehr. from the Colombian Amazonia. Revue d Hydrobiologie Tropicale 1994; 27(4):1-26

Correa HD, Ruiz SL, Arévalo LM, editores. 2005. Plan de acción en biodiversidad de la cuenca del Orinoco - Colombia / 2005 - 2015 - Propuesta Técnica. Bogotá D.C. Corporinoquia, Cormacarena, I.A.v.H, Unitrópico, Fundación Omacha, Fundación Horizonte Verde, Universidad Javeriana, Unillanos, WWF - Colombia, GTZ - Colombia.

Cortés A. 2004. Suelos Colombianos: Una mirada desde la academia. Colección Estudios Ambientales. Bogotá: Fundación Universidad de Bogotá Jorge Tadeo Lozano. Bogotá.

Cotner JB, Montoya JV, Roelke DL, Winemiller KO. Seasonally variable riverine production in the Venezuelan Ilanos. J. N. Am. Benthol. Soc, 2006; 25(1):171-184.

Cox EJ. 1996. Identification of freshwater diatoms from live material. Chapman \& Hall.

Croasdale H, Bicudo C, Prescott G. 1983. A synopsis of North American Desmids. Part II. Desmidiaceae: Placodermae. Section 5. University of Nebraska Press. 
Dezzeo N, Herrera R, Escalante G, Chacón N. Deposition of sediments during a flood event on seasonally flooded forests of the lower Orinoco River and two of its black-water tributaries, Venezuela. Biogeochemistry 2000; 49: 241-257.

Donato JC. Los sistemas acuáticos de Colombia: Síntesis y Revisión. Cuad Divulg PUI, 1991; 4:1-4.

Duque SR, Núñez M. Ficoflora de algunos ambientes acuaticos de la Amazonia Colombiana. Caldasia 1997; 19(1/2):279-284.

Duque SR. 1997. Tipificación limnológica de algunos lagos de la amazonia colombiana a través de la composición, biomasa y productividad del fitoplancton. Tesis Maestría en Ecología. Facultad de Ciencias. Universidad Nacional de Colombia. Bogotá.

Elmoor-Loureiro LM. 1997. Manual de identificação de cladóceros límnicos do Brasil. Brasilia: Editora Universia, Universidade Católica do Brasilis.

Galvis G, Mojica Jl,Rodríguez F. 1989. Estudio Ecológico de una laguna de desborde del Río Metica, Orinoquia Colombiana. Centro Editorial Universidad Nacional de Colombia, Fondo FEN Colombia. Bogotá.

Gómez MC. 2008. Dinámica espacial y temporal de la comunidad fitoplanctónica en el lago Yahuarcaca, planicie de inundación del río Amazonas. Tesis MSc. Universidad Javeriana. Bogotá.

González-González LM, Galindo T, Reyes C, Pérez J. Evaluación de dos cuerpos de agua lénticos mediante indicadores fitoplanctónicos y Contenido de Nutrientes (Puerto López, Meta Colombia). Memorias Seminario Colombiano de Limnología; 2006;1:73-83.

Hamilton SK, WM Lewis Jr. Causes of seasonality in the chemistry of a lake on the Orinoco River Floyd plain Venezuela. Limnol. Oceanogr. 1987; 32(6): 1277-1290.

Hamilton SK, WM Lewis Jr. Basin morphology in relation to Chemicals and ecological characteristics of lakes on the Orinoco River floodplain, Venezuela. Arch. Hydrobiol. 1990; 119 (4): 393-425

Legendre P, Legendre L. 1998. Numerical Ecology. Developments in Environmental Modelling 20. Elsevier, The Netherlands.

Lewis W, Saunders J, Dufford R. 1990. Suspended organisms and biological carbon flux along the lower Orinoco River. En: Weibezahn $\mathrm{FH}$, Alvarez $\mathrm{H}$, Lewis W, Editores. El Orinoco como un ecosistema. Primera Edición. Caracas- Venezuela, Editorial Galac S.A; 269-300.

Lewis WM Jr, Hamilton SK, Saunders III JF. 1995. Rivers of Northern South America. Pp. 219-256 In: Cushing \& Cummins (eds.) Ecosystems of the World: Rivers. Elsevier, NY.

Losada-Rodríguez LE. 1992. Estudio comparatico de la comunidad fitoplanctónica en las lagunas Menegua y Mateyuca en el municipio de Puerto López (Meta). Tesis de Grado. Departamento de Biología, Universidad Nacional de Colombia, Bogotá.

Marciales-Caro LJ. 2006. Ecología funcional del fitoplancton en dos lagos de la ribera colombiana del Río Mazonas (Tarapoto y el Correo). Tesis de pregrado, departamento de
Biología, Facultad de Ciencias, Universidad Nacional de Colombia, Bogotá.

Núñez-Avellaneda M, Duque S. 2001. Fitoplancton de algunos ríos y lagos de la Amazonía colombiana. En: Franky C, Zarate $\mathrm{C}$, Editores. Imani mundo: Estudios en la Amazonía colombiana. Universidad Nacional de Colombia- Unibiblos; P.305-335.

Núñez-Avellaneda M, Duque SR. Chlorococcales (Algae, Chlorophyceae) found in aquatic ennvironments of the Colombian Amazon basin. Caldasia; 1998; 20(1): 7-13.

Núñez-Avellaneda M. 2004. Fitoplancton de ecosistemas acuáticos de la cuenca del río Putumayo (Amazonía Colombo Peruana). Tesis Maestría en Estudios Amazónicos, Universidad Nacional de Colombia, Sede Amazonia. Leticia, Amazonas.

Paggi JC, Paggi JS. Zoopláncton de ambientes lóticos e lénticos do Río Paraná Médio. Acta Limnol Brasil, 1990; 3:685-719.

Palma L. 2011. Efecto de la conectividad del río Amazonas en la ecología del fitoplancton en lagos amazónicos. Tesis MSc en Estudios Amazónicos, Universidad Nacional de Colombia, sede Amazonia.

Pestalozzi HG. 1955. Das phytoplankton des sübwassers, Systematik und biologie 4. Teil. Euglenophyceen X. Klasse: Euglenophyceae. Stuttgart.

Prescott GW, Bicudo C, Vinyard W. 1982. A synopsis of north American desmids Part II. Desmidiaceae: Placoderme, Section 4. University of Nebraska Press.

Prescott GW, Croasdale H, Vinyard W. 1975. A synopsis of North American desmids. Part II. Desmidiaceae: Placoderme, section 1. University of Nebraska Press.

Prescott GW, Croasdale H, Vinyard W. 1977. A synopsis of North American desmids. Part II. Desmidiaceae: Placoderme, section 2. University of Nebraska Press.

Prescott GW, Croasdale H, Vinyard W, Bicudo, C. 1981. A synopsis of North American desmids. Part II: Desmidiaceae: Placoderme, section 3. University of Nebraska Press.

Ramírez-Gil H, Ajiaco-Martínez RH. 2001. La pesca en la baja Orinoquia colombiana: Una visión integral. INPA. Bogotá.

Rangel-CH O, Sánchez-C H, Lowy-C P, Aguilar-P M, Castillo-G A 2005. Región de la Orinoquia. En: Rangel CH O (Ed) 1995. Colombia Diversidad Biótica I. Instituto de Ciencias Naturales, Convenio Inderena-Universidad Nacional de Colombia. Bogotá, $442 \mathrm{p}$.

Reynolds CS. 2006. The Ecology of Phytoplankton. Cambridge University Press, Ecology, Biodiversity and conservation series, 535 pp.

Reynolds CS, Huszar V, Kruk C, Naselli-Flores L, Melo S. Towards a functional classification of the freshwater phytoplankton. Journal of Plankton Research 2002; 24: 417-428.

Rivera CA, Zapata AM, Pérez D, Morales Y, Ovalle H, Álvarez JP. Caracterización limnológica de humedales de la planicie de inundación del río Orinoco (Orinoquía, Colombia). Acta biol. Colomb. 2010; 15(1):145 - 166. 
Romero M, Galindo G, Armenteras D. 2004. Ecosistemas de la cuenca del Orinoco Colombiano. Instituto de Investigación de recursos Biológicos Alexandre von Humboldt. Bogotá, Colombia. 189 p.

Sala SE, Duque S, Núñez-Avellaneda M, Lamaro A. Diatoms from the Colombian Amazon: some species of the genus Eunotia (BaciIlariophyceae). Acta Amazónica 2002; 32(4):123-132.

San Jose JJ, Meirelles ML, Bracho R, Nikonova N. A Comparative analysis of the flooding and fire effects on the energy exchange in a wetland community (Morichal). J Hydrol; 2001; 242:228-245.

Sarmiento G, Pinillos M. Patterns and Processes in a Seasonally Flooded Tropical Plain: The Apure Llanos, Venezuela. Biogeogr. $2001 ; 28(8): 985-996$.

Saunders JF, Lewis WM. Zooplancton Abundance in the Caura River, Venezuela. Biotropica, 1998; 20(3):206-214.
Tell G, Conforti V. 1986. Euglenophyta pigmentadas de la Argentina. Berlin-Stuttgart. Ed. Cramer Bibl. Phycol. 75.

Torres-Bejarano A. 2006. Ecología funcional de fitoplancton Durante dos Periodos Hidrológicos en el sistema lagunar de Yahuarcaca (Amazonia Colombiana). Tesis Departamento de Biología, Universidad Pedagógica Nacional. Bogotá.

Twombly S, Lewis W. Zooplankton abundance and species composition in Laguna la Orsinera, a Venezuelan floodplain lake. Arch Hydrobiol, 1987; 79(1):87-107.

Weibezhan F. 1990. Hidroquímica y sólidos suspendidos en el Alto y Medio Orinoco. Pp 150-210. In: Weibezahn et al.. (Eds.). El Orinoco como un ecosistema. Primera Edición. Caracas- Venezuela, Editorial Galac S.A. 